\title{
SOME NONLINEAR DYNAMIC INEQUALITIES ON TIME SCALES
}

\section{S. H. SAKER}

Abstract. Our aim in this paper is to establish some explicit bounds of solutions of a certain class of nonlinear dynamic inequalities on a time scale $\mathbb{T}$ which is unbounded above. Also, we establish some sufficient conditions for global existence and an estimate of the rate of decay of the solutions. These on the one hand generalizes and on the other hand furnish a handy tool for the study of qualitative as well as quantitative properties of solutions of dynamic equations on time scales.

Mathematics subject classification (2010): 26D15, 26D20, 39A12.

Keywords and phrases: Dynamic equations, time scale, integral inequality.

\section{REFERENCES}

[1] D. A. Anderson, Young's integral inequality on time scales revisited, J. Ineq. Pure Appl. Math., 8, 3 (2007), Art. 64, 5 pp.

[2] D. A. Anderson, Nonlinear dynamic integral inequalities in two independent variables on time scale pairs, Advances Dyn. Syst. Appl., 3 (2008), 1-13.

[3] D. A. ANDERSon, Dynamic double integral inequalities in two independent variables on time scales, J. Math. Inq., 2 (2008), 163-184.

[4] M. Bohner And A. Peterson, Dynamic Equations on Time Scales: An Introduction with Applications, Birkhäuser, Boston, 2001.

[5] E. Akin-Bohner, M. Bohner, F. Akin, Pachpatte inequalities on time scales, JIPAM. J. Ineq. Pure Appl. Math., 6 (2005), 1-23.

[6] C. M. DAFERmos, The second law of thermodynamics and stability, Arch. Rational Mech. Anal., 70 (1979), 167-179.

[7] S. HILGER, Analysis on measure chains-a unified approach to continuous and discrete calculus, Results Math., 18 (1990), 18-56.

[8] V. KaC And P. Cheung, Quantum Calculus, Springer, New York, 2001.

[9] W. N. LI, Some Pachpatte type inequalities on time scales, Comp. Math. Appl., 57 (2009), 275-282.

[10] W. N. LI, Some new dynamic inequalities on time scales, J. Math. Anal. Appl., 319 (2007), 802-814.

[11] D. S. Mitrinović, J. E. PeČAriĆ And A. M. Fink, Classical and New Inequalities in Analysis, Kluwer Academic Publ., 1993.

[12] L. OU-IANG, The boundedness of solutions of linear differential equations $y^{\prime \prime}+A(t) y=0$, Shuxue Jinzhan, 3 (1957), 409-415.

[13] B. G. PACHPATTE, On some new inequalities related to certain inequalities in the theory of differential equations, J. Math. Anal. Appl., 189 (1995), 128-144.

[14] S. H. SAKER, Some nonlinear dynamic inequalities on time scales and applications, Journal of Math. Ineq., 4 (2010), 561-579.

[15] V. Spedding, Taming Nature's Numbers, New Scientist, July 19, 2003, 28-31. 